\title{
Water in Soybean Oil Microemulsions as Medium for Electrochemical Measurements
}

\author{
Carla R. B. Mendonça, Clara I. D. Bica and Clarisse M. S. Piatnicki*
}

Instituto de Química, Universidade Federal do Rio Grande do Sul, Av. Bento Gonçalves, 9500, CP 15003, 91501-970

Porto Alegre - RS, Brazil

\begin{abstract}
Microemulsões de água em óleo de soja (w/o ME) foram preparadas com dodecil sulfato de sódio (SDS) como surfactante e com os álcoois amílico ou isoamílico, como co-surfactantes. A composição 40,0\% de óleo, 43,2\% de álcool, 10,8\% de SDS e 6,0\% de água, em massa, na razão 1:4 [SDS]:[álcool], apresentou a maior estabilidade termodinâmica. O tamanho das gotículas e seu coeficiente de difusão nas microemulsões, $\mathrm{D}_{\mathrm{w} / \mathrm{o}}$, foram determinados por medidas de espalhamento de luz dinâmico (DLS). As reações de oxidação de ferroceno $(\mathrm{Fc})$ e de água, e a redução de ácido oleico foram observadas nas ME por voltametria em um ultramicroeletrodo (ume) de disco de Pt. Os valores de $\mathrm{D}_{\mathrm{w} / \mathrm{o}}$ determinados a partir de medidas eletroquímicas são menores do que os calculados a partir daquelas por DLS. Isto indica que a oxidação da água requer a difusão para o eletrodo tanto das gotículas como das moléculas de água nelas contidas. Os resultados evidenciam a possibilidade de determinação de analitos em óleos vegetais por métodos eletroanalíticos.
\end{abstract}

Microemulsions of water in soybean oil (w/o ME) were prepared with sodium dodecyl sulfate (SDS) as surfactant and amyl or isoamyl alcohol, as co-surfactants. Microemulsions containing $40.0 \%$ oil, $43.2 \%$ alcohol, $10.8 \%$ SDS and $6.0 \%$ water in weight, in the ratio 1:4 [SDS]:[alcohol] showed the highest thermodynamic stability. The aqueous droplet size and its diffusion coefficient $\mathrm{D}_{\mathrm{w} / \mathrm{o}}$ in the ME were determined through dynamic light scattering (DLS). Voltammetric measurements in the ME at a Pt disk ultramicroelectrode (ume) evidenced the oxidation of both water and ferrocene $(\mathrm{Fc})$, and the reduction of oleic acid. The $\mathrm{D}_{\mathrm{w} / \mathrm{o}}$ values calculated from the limiting current being lower than the ones obtained from DLS indicate that water oxidation probably requires diffusion towards the electrode of both the droplets and the water molecules from inside the droplets. The results show that electroanalytical determinations can be carried out in w/o ME.

Keywords: microemulsions, soybean oil, voltammetry, ferrocene, oleic acid

\section{Introduction}

Equilibria in water-oil interfaces have been studied in order to quantify solvent extraction as well as charge transfer processes. In this sense new analytical methodologies such as slow extrusion of water into a nonaqueous phase (and vice-versa) through a capillary, ${ }^{1}$ the use of micrometric electrodes ${ }^{2-3}$ and of microemulsions $s^{4,5}$ (ME) have been proposed recently. Besides a significant decrease in costs and toxicity due to the decrease in organic solvents consumption, ${ }^{6-8}$ water in oil ME allow to carry out electrochemical measurements due to the enhanced electrical conductivity imparted to the system by the presence of water.

Microemulsions are thermodynamically stable systems composed of water, oil and surfactant, and, in some cases,

\footnotetext{
* e-mail: clarisse@iq.ufrgs.br
}

an alcohol can be added as co-surfactant. ${ }^{9-12}$ Their behavior is similar to the one of polar organic solvents and, although apparently homogeneous under visual observation, ME are actually microheterogeneous (perhaps nanoheterogeneous would be a better term) systems formed of particles with diameters in the order of 5 to $100 \mathrm{~nm}$ suspended in a continuous phase..$^{8,13-15}$ The transparency showed by these systems is due to the fact that the size of the particles is much smaller than the wavelengths of radiation in the visible region. ${ }^{16}$

The conducting properties of ME are given mainly by the surfactant or by the addition of a supporting electrolyte. Due to the small interfacial tension, usually smaller than $0.001 \mathrm{dyn}^{-1}$, these systems are able to dissolve a high number of substances. ${ }^{7,14,16}$

The microstructure of ME has been elucidated by various techniques, as for example static and dynamic light scattering, dielectric relaxation, time-resolved fluorescence 
or phosphorescence quenching, electric field or temperature jump, small-angle neutron scattering (SANS) and small-angle X-ray scattering (SAXS). These techniques can afford fruitful information about the core and surface monolayer structure, the particle size distribution, the polydispersity, the droplet clustering and diffusion dynamics, the inter-droplet exchange rate and the fluidity of the monolayer. ${ }^{5,17,18}$

The rather classical methods of characterization through viscosity, interfacial tension, conductivity, turbidity and osmotic pressure measurements are still very reliable and informative. ${ }^{14,18-21}$ Particularly, the knowledge of the dynamic properties of the suspended droplets is very important in understanding the conductivity, chemical reactions and mass transfer processes occurring in these media. ${ }^{18}$

A stable homogeneous mixture is transparent, whereas an unstable not homogeneous mixture is turbid unless the components of the mixture have identical refractive indexes. The transparent to the turbid state can be brought about by variations of temperature, pressure or composition of the mixture and the transition point, the point of incipient phase separation, is the cloud point. ${ }^{22}$ When the sample reaches the cloud point temperature, the sample gets turbid and ceases to show the transparency which characterizes a $\mathrm{ME}$, a new phase starting to separate.

Electroanalytical techniques may provide valuable information concerning the structural evolution, interparticle interaction and transport data, ${ }^{17,23,24}$ yet there are very few studies employing electroanalytical methods in ME of water in vegetable oils. In this sense a Pt disk ultramicroelectrode (ume), allows to carry out linear voltammetric ${ }^{25}$ measurements in resistive media ${ }^{26}$ as is the case in this study. For these small sized electrodes the limiting current expression is:

$\mathrm{i}_{\lim }=4 n \mathrm{FrDC}$

where $n$ is the number of electrons, F the Faraday constant, $\mathrm{r}$ the radius of the $\mathrm{Pt}$ disk in $\mathrm{cm}, \mathrm{C}$ the concentration of the electroactive species in mol $\mathrm{cm}^{-3}$ and $\mathrm{D}$ the diffusion coefficient of the electroactive species in $\mathrm{cm}^{2} \mathrm{~s}^{-1}$. Then the $\mathrm{i}_{\lim }$ unity will be the Ampere.

This study aims to prepare, to characterize and to evaluate the experimental conditions allowing to carry out electroanalytical methods in (w/o) ME applied to the quantitative determination of analites such as phenols, free fatty acids, tocopherols, etc. in vegetable oils..$^{27,28}$ Since these species constitute minor components in edible oils, both a maximum amount of oil in the ME is desirable and the standard addition calibration technique should be employed, in order to increase the analysis sensitivity.

\section{Experimental}

\section{Materials}

Refined and deodorized soybean oil stored in metallic containers was obtained from the local market. The ME were prepared in different ways, employing as surfactant 99\% sodium dodecyl sulfate (SDS) from Merck, Milli-Q water previously distilled and, alternatively, $95 \%$ ethyl alcohol from Synth, 99\% amyl, isoamyl and isobutyl alcohols from Merck and 96\% hexyl alcohol from Riedel as co-surfactants. Pure oleic acid from Reagen and ferrocene 98\% from Fluka were employed, respectively, as a probe analite and as an electrochemical reference material, ${ }^{29,30}$ the voltammograms being obtained with an EG\&G PAR Model 264A potentiostat. As for conductivity measurements they were carried out with an Oakton 35607 $10 \mathrm{~A}$ instrument.

Light scattering measurements were performed with a Spectra-Physics model 170 spectrogoniometer from Brookhaven Instruments using a $\lambda=632.8 \mathrm{~nm} \mathrm{He}-\mathrm{Ne}$ laser while viscosity and refractive index values were obtained respectively in a Brookfield LVDV-II+ instrument and an Abbé refractometer. Moreover, a Shimadzu UV-1601PC UV-visible spectrometer was employed to obtain the absorbance spectrum of microemulsions in the absence and in the presence of dissolved ferrocene $(\mathrm{Fc})$.

\section{Methods}

Microemulsion preparation. Microemulsions were prepared by mixing the surfactant (SDS) with an alcohol co-surfactant and water. The oil was then progressively added to the mixture, in steps of $10 \%$ increase in the mass of the mixture, covering an oil composition range from 10 to $90 \%$. Several alcohols were tested as co-surfactants, namely ethyl, amyl, isoamyl, isobutyl and hexyl alcohol. The mixtures were stirred for 1 hour with a GFL 3006 magnetic shaker and the oil additions were performed within five-minute intervals. The surfactant to co-surfactant ratio was varied from 1:1 up to 1:6 in successive addition steps of one part of the co-surfactant. For ratios resulting in transparent ME, experiments with addition steps of 0.1 part of co-surfactant were also performed.

In order to evaluate the repeatability of the systems, all characterization data were obtained from triplicates of each ME composition.

Microemulsion characterization. Intensities of DLS measurements were correlated with a 264-channel BI-9000 AT instrument covering 7 decades in delay time. The samples were in a refractive-index-matching liquid 
(decaline). In order to characterize the hydrodynamic behavior of the suspended particles the multisampling time autocorrelation functions were analyzed by oneexponential fit (Microcal Origin ${ }^{\circledR}$ 6.0) and by inverse Laplace transformation using the $\mathrm{CONTIN}^{31}$ program.

The DLS experiments were carried out at $25.0 \pm 0.5^{\circ} \mathrm{C}$ and at a fixed scattering angle of $90^{\circ}$ except in diffusion coefficient determinations of water droplets in the presence and in the absence of dissolved $\mathrm{Fc}$ for which the scattering angles were $35 ; 45 ; 55 ; 65 ; 75 ; 85 ; 90 ; 105 ; 115 ; 125$ and $135^{\circ}$. The UV-Visible spectra of soybean oil and of microemulsions with and without dissolved Fc were obtained in order to make sure that no light absorption occurs in the wavelength of the He-Ne laser used in light scattering experiments.

In order to evaluate the thermodynamic stability of the microemulsions, cloud point measurements were performed by visual inspection in the temperature range from 9 to $70{ }^{\circ} \mathrm{C}$ in steps of 3 degrees up to $25^{\circ} \mathrm{C}$ and afterwards in steps of 5 degrees, for $5 \mathrm{~min}$, in a thermostated bath from Lauda. As for the refractive index measurements, they were carried on at $21.0 \pm 0.5^{\circ} \mathrm{C}$ while viscosity ones were performed at $25.0 \pm 0.1^{\circ} \mathrm{C}$.

Prior to all measurements the samples were centrifuged during $30 \mathrm{~min}$ at $4000 \mathrm{rpm}$ in order to minimize the interference of dust particles.

Electrochemical measurements. A standard $\mathrm{KCl}$ solution, whose specific conductivity is $1.412 \mu \mathrm{S} \mathrm{cm}^{-1}$ at $25{ }^{\circ} \mathrm{C}$, was employed in conductivity measurements carried out at $21.0 \pm 0.5^{\circ} \mathrm{C}$. Current-potential curves in ME were obtained at room temperature, the electrochemical cell consisting of a three-electrode assembly with a $1 \mu \mathrm{m}$ radius $\mathrm{Pt}$ disk working ume, a reference $\mathrm{AgCl} / \mathrm{Ag}$ electrode and a Pt auxiliary one. Unless indicated, the majority of the results are referred to the $\mathrm{AgCl} / \mathrm{Ag}$ reference electrode while in some experiments a Pt quasi-reference ${ }^{32}$ has been employed.

In order to have reproducible results, the working electrode was cleaned before each measurement by immersion for 5 minutes, respectively, in acetone and in 1:1 sulfuric acid/water and finally ultrasonicated in water.

For the determination of the ME electrochemical domain the Pt ume was swept from $0.00 \mathrm{~V}$ to $3.00 \mathrm{~V}$ and from $0.00 \mathrm{~V}$ to $-2.50 \mathrm{~V}$ at $1 \mathrm{mV} \mathrm{s}^{-1}$, the anodic and cathodic limits being arbitrarily defined as the potential at which the current density reached $0.1 \mathrm{~A} \mathrm{~cm}^{-2}$.

The anodic linear potential scan of the Pt ume in the $\mathrm{ME}$ in the absence and in the presence of Fc 20, 30 and 40 $10^{-3} \mathrm{~mol} \mathrm{~L}^{-1}$ was carried out under $\mathrm{N}_{2}$ flux, the potential being swept at $1 \mathrm{mV} \mathrm{s}^{-1}$. As for the electrochemical behavior of oleic acid, which was added to the ME in the concentrations 30,50 and $7010^{-3} \mathrm{~mol} \mathrm{~L}^{-1}$, it was analyzed by linear voltammetry at a Pt ume, under $\mathrm{N}_{2}$ flux, in both the anodic and cathodic regions, the potential being swept at $2 \mathrm{mV} \mathrm{s}^{-1}$.

\section{Results and Discussion}

\section{Microemulsion formation}

The ME formation was evidenced through its visual transparency. Among the co-surfactants tested in the experiments, amyl and isoamyl alcohol lead to very stable $\mathrm{ME}$, the best surfactant to co-surfactant ratio ranging between 1:3 and 1:4.

As it has been said before, a maximum amount of oil in the $\mathrm{ME}$ is desirable in order to increase the analysis sensitivity. The limiting amount of oil in the microemulsions is $40 \%$ of the total mass, higher oil fractions leading to their thermodynamic instability as will be described bellow.

Pseudo-ternary phase diagrams similar to the one in Figure 1 were obtained for ME prepared with several water/ oil/surfactant mixtures containing alternatively amyl or isoamyl alcohol as co-surfactant. It can be seen that the microemulsion composition chosen for the experimental measurements is located in a limiting region of thermodynamic stability.

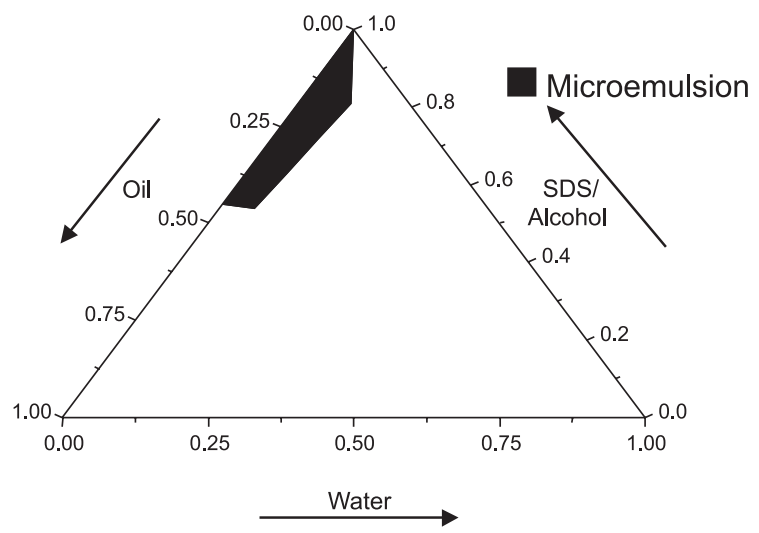

Figure 1. Phase diagram of water/ oil/ SDS and co-surfactant mixtures at $25{ }^{\circ} \mathrm{C}$. The shaded area indicates the microemulsions domain.

\section{Cloud point}

Microemulsions of water in soybean oil loose their thermodynamic stability at low temperatures. Figures $2 \mathrm{a}$ and $2 \mathrm{~b}$ show, respectively, the cloud points obtained for the microemulsions prepared with amyl or isoamyl alcohol. Both $\mathrm{ME}$ are stable up to $70{ }^{\circ} \mathrm{C}$ while temperatures under 
around $10^{\circ} \mathrm{C}$ lead to the instability of the system. As it can be seen in Figures $2 \mathrm{a}$ and $2 \mathrm{~b}$ the microemulsions prepared with isoamyl and amyl alcohol in the ratio $1: 4$ [SDS]:[alcohol] presented a similar behavior, their cloud point being at $9^{\circ} \mathrm{C}$.
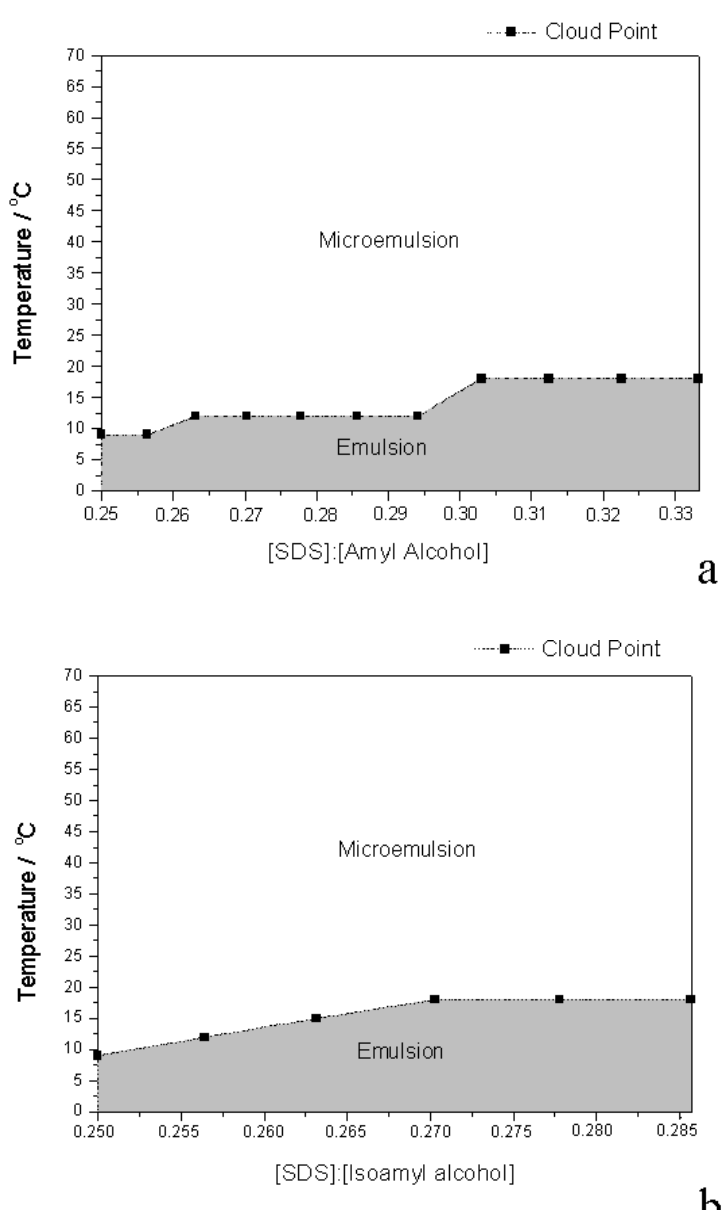

Figure 2. Cloud point of microemulsions prepared with amyl (a) and isoamyl (b) alcohol, for several alcohol to surfactant proportions, $\mathrm{m} / \mathrm{m}$.

It is observed that the higher thermodynamic stability values were found for ME prepared with amyl or isoamyl alcohol in the ratio 1:4 [SDS]:[alcohol] with the composition $40.0 \%$ oil, $43.2 \%$ alcohol, $10.8 \%$ SDS and $6.0 \%$ water, $\mathrm{m} / \mathrm{m}$. Therefore, these systems were chosen to perform the diffusion coefficient, conductivity and electrochemical measurements, all reported results referring to them, unless otherwise stated.

\section{Dynamic Light Scattering}

Among the spectroscopic techniques available to probe microheterogeneous systems, particularly microemulsions, ${ }^{5}$ scattering ones are the most versatile and non- destructive. The Brownian motion of particles in a medium causes the scattered light intensity to fluctuate strongly. In a pure liquid light is scattered due to density fluctuations whereas in a solution scattering is also due to concentration fluctuations. ${ }^{8}$ On the other hand, by analyzing the fluctuations in the intensity of scattered light, DLS gives information on the Brownian motion of the particles.

In DLS the scattered light intensity autocorrelation function $\mathrm{G}_{2}(\tau)$ is the measured parameter:

$\mathrm{G}_{2}(\tau)=<\mathrm{I}(0) \mathrm{I}(\tau)>$

which may be normalized through the base line $\langle\mathrm{I}\rangle^{2}$ to give:

$\mathrm{g}_{2}(\tau)=\mathrm{G}_{2}(\tau) /<\mathrm{I}>^{2}$

where $\tau$ is the delay time.

If the fluctuations in the scattered field show a Gaussian distribution, the normalized intensity autocorrelation function $\mathrm{g}_{2}(\tau)$ may be related to the field correlation function $\mathrm{g}_{1}(\tau)$ by the so-called Siegert relationship:

$\mathrm{g}_{2}(\tau)=1+\beta\left[\mathrm{g}_{1}(\tau)\right]^{2}$

where $\beta$ is called the optical coherence factor which depends only on the experimental optical set-up. In the case of a monodisperse ideal system, $\mathrm{g}_{1}(\tau)$ is represented as a single exponential:

$\mathrm{g}_{1}(\tau)=\exp \left(-\mathrm{Dq}^{2} \tau\right)$

where $\mathrm{D}$ is the translational diffusion coefficient and $\mathrm{q}$ is the magnitude of the scattering vector

$\mathrm{q}=(4 \pi \mathrm{n} / \lambda) \sin \theta / 2$

defined in terms of the refractive index of the medium, $\mathrm{n}$, the radiation wavelength in vacuum, $\lambda$, and the scattering angle, $\theta$. The inverse of q represents the observable length scale of the scattering object.

When the system is polydisperse, which is the usual case, the field correlation function must be described by a weighed sum of exponentials:

$g_{1}(\tau)=\int_{0}^{\infty} G(\Gamma) \exp (-\Gamma \tau) d \Gamma$

where $G(\Gamma)$ is the distribution function of the relaxation rates.

One of the methods which can be applied to analyze the function given by equation (7) is the FORTRAN program 
CONTIN.$^{31}$ This program determines $\mathrm{G}(\Gamma)$ by performing an inverse Laplace transform on equation (7). ${ }^{8,33,34}$

Figure 3 shows time correlation functions well fitted through a single exponential for microemulsions containing either isoamyl or amyl alcohol as co-surfactant.

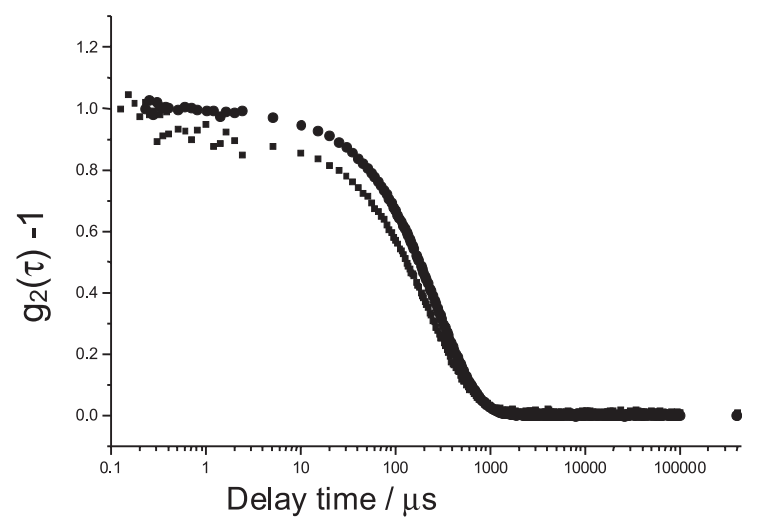

Figure 3. Time correlation function for microemulsions containing $40.0 \%$ oil, $43.2 \%$ alcohol, $10.8 \%$ SDS, and $6.0 \%$ water, (O) with amyl alcohol, ( $\mathbf{\square}$ ) with isoamyl alcohol, at a scattering angle $\theta=90^{\circ}$.

The hydrodynamic radius ( $\mathrm{Rh}$ ) obtained by using the CONTIN program gives the water droplets size in the ME. The average $\mathrm{Rh}$ values for ME prepared with isoamyl and amyl alcohol were, respectively, $3.2 \pm 0.1 \mathrm{~nm}$ and $4.3 \pm 0.2$ $\mathrm{nm}$ and they did not show significant changes with the variation of the scattering angle (data not shown).

The smaller hydrodynamic radius for droplets in $\mathrm{ME}$ prepared with isoamyl alcohol may have two origins: one is related to steric effects and the other to the continuous phase polarity. For microemulsions prepared, respectively, with the same amount of amyl or isoamyl alcohol, the amount of the latter dissolved in the interfacial region is probably smaller than in the case of amyl alcohol, due to its more bulky chain end. Therefore, both the isoamyl alcohol concentration in the oil phase and consequently the polarity of the continuous phase are higher for $\mathrm{ME}$ containing this alcohol leading to a decrease in the water core. ${ }^{35}$

Table 1 presents the characterization results for the w/o ME containing either amyl or isoamyl alcohol as cosurfactants. Using the CONTIN program, a narrow particle size distribution was observed, approximately $98 \%$ of the particles lying in the same $\mathrm{Rh}$ range. This evidence has been confirmed by the single exponential fit to the correlation functions showing that these ME are monodispersed systems.

The refractive index value of microemulsions is slightly lower than the one for soybean oil $\left(\mathrm{n}_{\text {soy oil }}=1.475\right)$ while the viscosity value, as expected, shows a significant decrease $\left(\eta_{\text {soy oil }}=52.0 \mathrm{cP}\right)$.

\section{Translational diffusion coefficient}

Relaxation rate measurements as a function of the square of the scattering vector (data not shown) allowed the determination of the diffusion coefficient of aqueous droplets in $\mathrm{ME}\left(\mathrm{D}_{\mathrm{w} / \mathrm{o}}\right)$ the values of which are, respectively $6.6 \times 10^{-8} \mathrm{~cm}^{2} \mathrm{~s}^{-1}$ in isoamyl alcohol and $5.4 \times 10^{-8} \mathrm{~cm}^{2} \mathrm{~s}^{-1}$ in amyl alcohol (see Table 2). The results have shown that the droplet diffusion is translational and that there is no influence of a rotational movement.

Moreover, the aim of this study being to perform electrochemical measurements in water in soybean oil ME, Fc was added to this medium in order to evaluate its influence in the $\mathrm{D}_{\mathrm{w} / \mathrm{o}}$ value obtained from DLS data. Since Fc dissolves preferentially in the oil phase, neither its presence nor its concentration affected significantly the $\mathrm{D}_{\mathrm{w} / \mathrm{o}}$ values, the small deviations observed in the values in Table 2 being attributed to experimental errors.

Table 1. Physico-chemical characterization data for $40.0 \%$ oil, $43.2 \%$ alcohol, $10.8 \%$ SDS and $6.0 \%$ water microemulsions

\begin{tabular}{|c|c|c|c|c|}
\hline Microemulsions & {$[\mathrm{SDS}] /[$ alcohol $]$} & $\mathrm{Rh}^{\mathrm{a}}(\mathrm{nm} \pm \sigma)$ & Refractive index $\pm \sigma$ & Viscosity $(\mathrm{cP} \pm \sigma)$ \\
\hline w/o SDS + amyl alcohol & $1: 4.0$ & $4.3 \pm 0.2$ & $1.436 \pm 0.01$ & $14.0 \pm 0.2$ \\
\hline w/o SDS + isoamyl alcohol & $1: 4.0$ & $3.2 \pm 0.1$ & $1.435 \pm 0.01$ & $14.2 \pm 0.1$ \\
\hline
\end{tabular}

${ }^{a}$ Hydrodynamic radius obtained by CONTIN program; $\sigma$ - standard deviation.

Table 2. Diffusion coefficient values $\left(\mathrm{D}_{\mathrm{w} / \mathrm{o}}\right)$ from DLS measurements for $40.0 \%$ oil, $43.2 \%$ alcohol, $10.8 \%$ SDS and $6.0 \%$ water microemulsions

\begin{tabular}{lcccc}
\hline & & \multicolumn{2}{c}{$\mathrm{D}_{\text {w/o }}\left(\mathrm{cm}^{2} \mathrm{~s}^{-1}\right)$} & \\
Microemulsions & Without Fc & $10 \times 10^{-3} \mathrm{~mol} \mathrm{~L}^{-1} \mathrm{Fc}$ & $30 \times 10^{-3} \mathrm{~mol} \mathrm{~L}^{-1} \mathrm{Fc}$ & $50 \times 10^{-3} \mathrm{~mol} \mathrm{~L}^{-1} \mathrm{Fc}$ \\
\hline w/o SDS + amyl alcohol & $5.4 \times 10^{-8}$ & $5.0 \times 10^{-8}$ & $5.0 \times 10^{-8}$ & $5.8 \times 10^{-8}$ \\
w/o SDS + isoamyl alcohol & $6.6 \times 10^{-8}$ & $6.9 \times 10^{-8}$ & $6.6 \times 10^{-8}$ & $6.3 \times 10^{-8}$ \\
\hline
\end{tabular}




\section{Molecular absorption}

Molecular absorption measurements performed in the range from 400 to $800 \mathrm{~nm}$ indicated that soybean oil and microemulsions absorb light below $450 \mathrm{~nm}$ while the ME containing ferrocene $50 \times 10^{-3} \mathrm{~mol} \mathrm{~L}^{-1}$ absorbs in the region from 400 to $530 \mathrm{~nm}$ with an absorption peak at $480 \mathrm{~nm}$. Therefore, there is no interference on DLS results from Fc light absorption at $633 \mathrm{~nm}$, which is the wavelength employed for light scattering measurements (Figure 4).

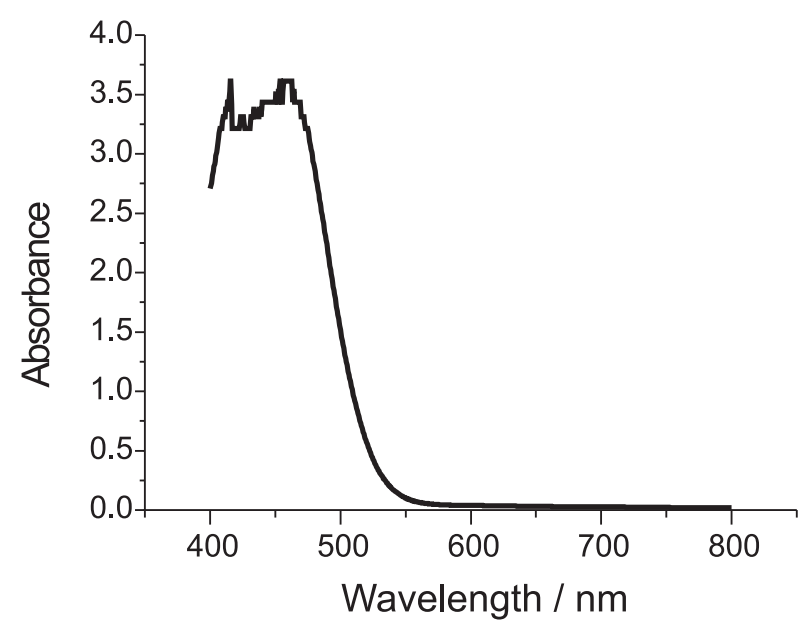

Figure 4. UV-Vis spectrum for microemulsion containing $40.0 \%$ oil, $43.2 \%$ alcohol, $10.8 \%$ SDS, $6.0 \%$ water and $5010^{-3} \mathrm{~mol} \mathrm{~L}^{-1}$ ferrocene.

\section{Electrochemical measurements}

Data on electrochemical measurements carried out in w/o microemulsions are shown in Table 3. It is observed that the conductivity values are above 1000 times higher than the $0.02 \mu \mathrm{S} \mathrm{cm}^{-1}$ value measured for soybean oil and around 100 and 20 times higher than the $0.24 \mu \mathrm{S} \mathrm{cm}^{-1}$ and $1.37 \mu \mathrm{S} \mathrm{cm}^{-1}$ values measured for deionized and distilled water, respectively.

These results are in accordance with data in the literature, that attribute the conductivity of a water-in-oil microemulsion mainly to the fact that nanodroplets carry positive or negative excess charges. ${ }^{36}$ Charged droplets are formed by spontaneous number fluctuations of the ions residing on the droplets. The magnitude of these fluctuations is directly related to the Coulomb energy that is required to charge up a droplet.

The wave in the w/o ME at $\mathrm{E}_{1 / 2}$ around 1.2 V (see Figure 6 , curve a) is attributed to water oxidation since it was observed experimentally that the limiting anodic current increases with the water content in the ME. Moreover, this is the expected potential value for water oxidation at a $\mathrm{Pt}$ electrode vs. $\mathrm{AgCl} / \mathrm{Ag}$. On the other hand, in the cathodic region, a small wave is observed at around $-0.60 \mathrm{~V}$ and at $-1.20 \mathrm{~V}$ a rapid increase in current occurs, probably due to water reduction. When a Pt quasi-reference electrode is employed, the $E_{1 / 2}$ potential for water oxidation and the limiting potential attributed to its reduction are, respectively, $0.90 \mathrm{~V}$ and $-1.30 \mathrm{~V}$.

Therefore, following the arbitrarily chosen value of $0.1 \mathrm{~A} \mathrm{~cm}^{-2}$ for the current density, the electrochemical window for this medium is in the potential range from approximately -1.20 to $2.00 \mathrm{~V}$ vs. $\mathrm{AgCl} / \mathrm{Ag}$.

In viscous flow, the solute molecules or ions distort the streamlines, introducing a rotational quality to the previously irrotational flow; in conductance and diffusion, they lengthen the effective paths of the moving particles. The hydrodynamic theory of a liquid containing suspended or dissolved particles that cause a change in the local viscosity, falling off with an inverse power of distance from the particle has been discussed earlier. ${ }^{37}$ However, this discussion surmounts the scope of the present work.

The calculated water concentration obtained from mass and volume measurements of $\mathrm{ME}$ is $3 \mathrm{~mol} \mathrm{~L}^{-1}$ and the $\mathrm{D}_{\text {w/o }}$ coefficient values for water droplets (see Table 3 ) obtained from voltammetric experiments (water oxidation), were calculated using equation (1). It is seen that the ratios between $\mathrm{D}_{\mathrm{w} / \mathrm{o}}$ values obtained from DLS measurements to those calculated from equation (1) are, respectively, around 4 (ME containing amyl alcohol) and 7 (ME containing isoamyl alcohol).

Assuming that the diffusion coefficient value for species in the droplets is the same as the one of the

Table 3. Electrochemical data for microemulsions containing $40.0 \%$ oil, $43.2 \%$ alcohol, $10.8 \%$ SDS and $6.0 \%$ water. Conductivity, limiting anodic and cathodic and $\mathrm{E}_{1 / 2}$ potentials for oxidation at a Pt ume of $3 \mathrm{~mol} \mathrm{~L}^{-1} \mathrm{H}_{2} \mathrm{O}$ without $\mathrm{Fc}$ and with $\mathrm{Fc} 2010^{-3} \mathrm{~mol} \mathrm{~L}^{-1}, \mathrm{D}_{\mathrm{w} / \mathrm{o}}$ and $\mathrm{D}_{\mathrm{Fc}}$ calculated from equation (1)

\begin{tabular}{|c|c|c|c|c|c|c|c|}
\hline Microemulsion & $\begin{array}{l}\text { Conductivity } \\
\left(\mathrm{mS} \mathrm{cm}^{-1}\right)\end{array}$ & $\begin{array}{l}{ }^{a} \text { Anodic } \\
\text { limit (V) }\end{array}$ & $\begin{array}{l}{ }^{\mathrm{a} C a t h o d i c} \\
\text { limit (V) }\end{array}$ & $\begin{array}{c}{ }^{\mathrm{b}} \mathrm{E}_{1 / 2 \mathrm{w} / \mathrm{o}} \\
(\mathrm{V})\end{array}$ & $\begin{array}{l}{ }^{a} E_{1 / 2} F c \\
(V)\end{array}$ & $\begin{array}{c}{ }^{b} D_{w / o} \\
\left(\mathrm{~cm}^{2} \mathrm{~s}^{-1}\right)\end{array}$ & $\begin{array}{c}{ }^{a} D_{\mathrm{Fc}} \\
\left(\mathrm{cm}^{2} \mathrm{~s}^{-1}\right)\end{array}$ \\
\hline w/o SDS + amyl alcohol & 30.95 & 2.04 & -1.18 & 0.90 & 0.57 & $1.42 \times 10^{-8}$ & $5.2 \times 10^{-7}$ \\
\hline w/o SDS + isoamyl alcohol & 21.97 & 2.00 & -1.23 & 0.97 & 0.56 & $0.90 \times 10^{-8}$ & $5.1 \times 10^{-7}$ \\
\hline
\end{tabular}

${ }^{\mathrm{a}} \mathrm{AgCl} / \mathrm{Ag}$ reference electrode; ${ }^{\mathrm{b}} \mathrm{Pt}$ quasi-reference electrode. 
droplets $^{23}$ these results may be explained, in part, by the net positive charge carried by a fraction of the droplets as discussed above. ${ }^{36}$ Consequently, its transport to the surface of the electrode is hindered by an unfavorable migration component. Moreover, water molecules must break the nonpolar barrier around the droplets and diffuse to the surface of the electrode before the charge transfer reaction takes place. $^{23}$

In the cathodic region, water reduction should be facilitated by a migration component towards the electrode due to the net positive charge located into the water droplets, which can explain that no reduction wave is observed in the experimental conditions of this study. On the other hand, assuming that for the water reduction reaction the diffusion coefficient value $\mathrm{D}_{\mathrm{w} / \mathrm{o}}$ will be closer to the one found from DLS measurements, the limiting current will be theoretically around $6 \mathrm{nA}$, calculated from equation (1) using $\mathrm{D}_{\mathrm{w} / \mathrm{o}}=5.4 \times 10^{-8} \mathrm{~cm}^{2} \mathrm{~s}^{-1}$ (see Table 2). This hypothesis will be investigated in the sequence of this study.

Linear voltammetric measurements of deaerated ME, with and without dissolved Fc, were carried out in the anodic region at $1 \mathrm{mV} \mathrm{s}^{-1}$ using a Pt ume, SDS being the only electrolyte in the medium.

The voltammograms of ME containing 0, 20, 30 and $40 \times 10^{-3} \mathrm{~mol} \mathrm{~L}^{-1}$ of Fc and amyl alcohol as co-surfactant, are shown in Figure 5. The wave at $\mathrm{E}_{1 / 2}=0.50 \mathrm{~V} v s$. Pt quasi-reference electrode is attributed to the Fc oxidation, since it appears only when this compound is dissolved in the ME, the limiting current varying linearly with Fc concentration. The oxidation potential of $\mathrm{Fc}$ in the $\mathrm{ME}$ is therefore more anodic than in acetonitrile $\left(\mathrm{E}^{\mathrm{o}}=0.415 \mathrm{~V}\right.$ $v s$. standard calomel electrode, SCE) and in water $\left(\mathrm{E}^{\circ}=0.163 \mathrm{~V}\right.$ vs. SCE). ${ }^{24}$ On the other hand, as expected, the voltammogram in the cathodic region is not altered by the presence of Fc.

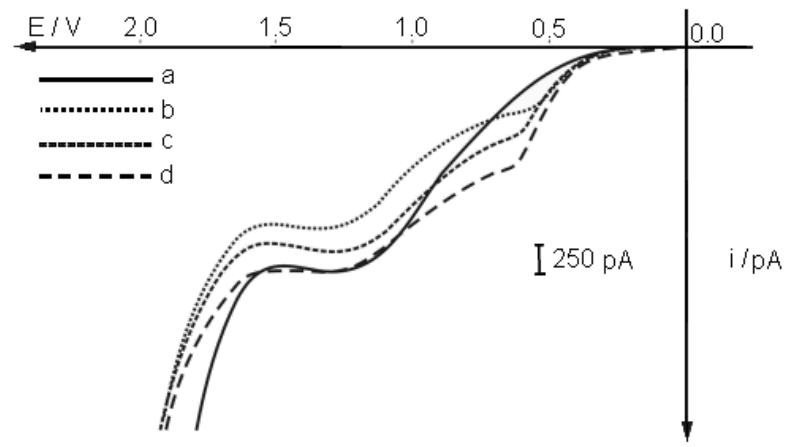

Figure 5. Voltammogram of microemulsions containing $40.0 \%$ oil, $43.2 \%$ amyl alcohol, $10.8 \%$ SDS and $6.0 \%$ water, (a) without ferrocene and (b) with $20 \times 10^{-3} \mathrm{~mol} \mathrm{~L}^{-1}$ ferrocene; (c) with $30 \times 10^{-3} \mathrm{~mol} \mathrm{~L}^{-1}$; (d) with $40 \times 10^{-3} \mathrm{~mol} \mathrm{~L}^{-1}$. Working Pt ume, Pt quasi-reference electrode and $\mathrm{v}=1 \mathrm{mV} \mathrm{s}^{-1}$.
By comparing the diffusion coefficient values for Fc oxidation from the oil phase and for water from droplets in the ME, it is observed that $\mathrm{D}_{\mathrm{Fc}}$ is, respectively, 60 and 40 times higher than $\mathrm{D}_{\mathrm{w} / \mathrm{o}}$ for $\mathrm{ME}$ containing, respectively, isoamyl and amyl alcohol. Considering that water droplets are surrounded by a film of nonpolar tails of dodecyl sulfate anions and amyl or isoamyl chains, ${ }^{14}$ its diffusion through the continuous oil medium must be slower than the one for $\mathrm{Fc}$ molecules. On the other hand, the net positive charge in the water droplets ${ }^{36}$ is probably the reason for the decrease in the limiting current observed for water oxidation in the presence of Fc (see Figure 5). The ferricinium ion, $\mathrm{Fc}^{+}$, present near the electrode hinders the migration of positive charged droplets and consequently the water oxidation reaction rate decreases, this difficulty being expected to increase with Fc concentration.

Therefore, the diffusion $\mathrm{D}_{\mathrm{w} / \mathrm{o}}$ value obtained from water oxidation experiments seems to include the droplets diffusion and the water molecules diffusion from inside the droplets to the surface of the electrode. ${ }^{23}$ This hypothesis could also explain the diffusion coefficient value found for $\mathrm{Fc}$ in $\mathrm{ME}$, higher than the value found for $\mathrm{D}_{\mathrm{w} / \mathrm{o}}$, in spite of the Fc much lower concentration.

The voltammograms of a ME without and with added oleic acid in the concentrations 30,50 and $7010^{-3} \mathrm{~mol} \mathrm{~L}^{-1}$ and amyl alcohol as co-surfactant were carried out at $2 \mathrm{mV} \mathrm{s}^{-1}$ using a Pt ume in deaerated medium (Figure 6).

The wave at $\mathrm{E}_{1 / 2}$ around $-0.60 \mathrm{~V}$ is attributed to oleic acid reduction, since the limiting current increases linearly with its concentration in the ME. It is also observed that $\mathrm{E}_{1 / 2}$ is slowly displaced to more negative potentials as the fatty acid concentration increases.

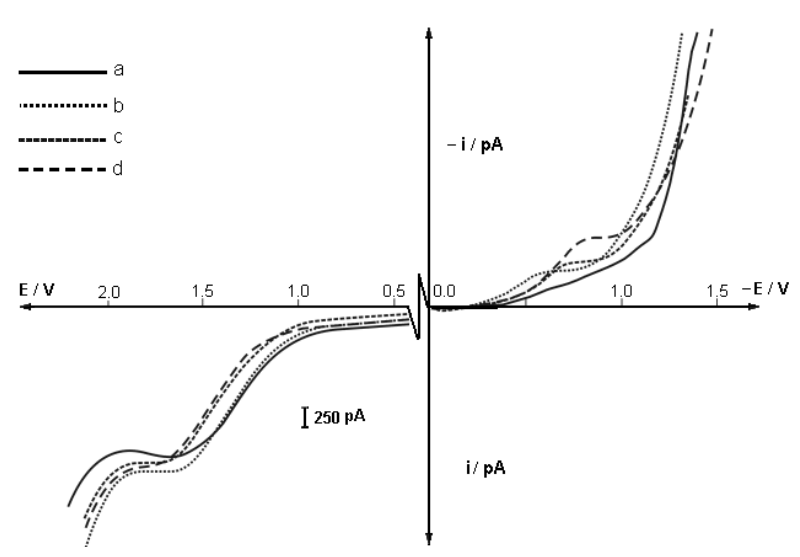

Figure 6. Voltammogram of microemulsions containing $40.0 \%$ oil, $43.2 \%$ amyl alcohol, $10.8 \%$ SDS and $6.0 \%$ water, (a) without oleic acid; (b) with $30 \times 10^{-3} \mathrm{~mol} \mathrm{~L}^{-1}$ oleic acid, (c) with $50 \times 10^{-3} \mathrm{~mol} \mathrm{~L}^{-1}$; (d) with $70 \times 10^{-3} \mathrm{~mol} \mathrm{~L}^{-1}$ oleic acid. Working $\mathrm{Pt}$ ume, $\mathrm{AgCl} / \mathrm{Ag}$ reference electrode and $\mathrm{v}=2 \mathrm{mV} \mathrm{s}^{-1}$. 
Fatty acids electroreduction is actually the reduction of hydrogen from the carboxylic group, forming the anion carboxylate..$^{38}$ On the other hand, no additional oxidation wave appears in the anodic region in the presence of oleic acid and no significant change in the limiting current of water oxidation is observed. However, a slight displacement to more positive potentials of the $\mathrm{E}_{1 / 2}$ values for water oxidation indicates, as well as the $\mathrm{E}_{1 / 2}$ displacement in the cathodic reaction, a decrease in the electrical conductivity of the ME. These results confirm data in the literature that oxidation of fatty acids at a Pt electrode occurs only at high-density currents $\left(>1 \mathrm{~A} \mathrm{~cm}^{-2}\right) .38$

The small reduction wave observed at $-0.60 \mathrm{~V}$ for the ME without added oleic acid (see Figure 6) indicates the presence of low quantities of free unsaturated fatty acids (mostly oleic, linoleic, linolenic and palmitic) ${ }^{39}$ in soybean oil.

Therefore, the reported results indicate that electroanalytical methods using ume allow the quantitative determination of species in water in soybean oil microemulsions.

\section{Conclusions}

Microemulsions of water in soybean oil containing either isoamyl or amyl alcohol present droplets of nanometric dimension and are stable up to $70{ }^{\circ} \mathrm{C}$ while temperatures under around $10^{\circ} \mathrm{C}$ lead to the instability of the systems. The droplets showed a translational diffusion character, no influence of a rotational movement being detected. On the other hand, the smaller hydrodynamic radius of the droplets in ME prepared with isoamyl alcohol evidences the influence of steric effects in the droplet/oil interface.

Electrical conductivity values higher than the ones for soybean oil and deionized water, and an electrochemical window of approximately $3 \mathrm{~V}$ were found for both isoamyl and amyl alcohol ME in the experimental conditions of this study.

The lower $\mathrm{D}_{\mathrm{w} / \mathrm{o}}$ values obtained from electrochemical measurements show that the transport of water molecules to the surface of the electrode is probably hindered, simultaneously, by an unfavorable migration component, the nonpolar barrier around the droplets and the diffusion of water molecules from inside the droplets to the surface of the electrode, before the charge transfer reaction may take place.

Also related to the unfavorable migration of the droplets is the lower oxidation rate for water molecules in the presence of $\mathrm{Fc}$, which is enhanced by the increase of $\mathrm{Fc}^{+}$ concentration near the electrode, thus evidencing further the presence of a positive charge in the ME droplets.

Finally, the linear dependence with concentration of the anodic and the cathodic limiting currents, respectively, in $\mathrm{Fc}$ oxidation and in oleic acid reduction, indicate that quantitative electroanalytical determinations may be carried out in water in soybean oil microemulsions.

\section{Acknowledgements}

The authors thank the support of CAPES.

\section{References}

1. Baars, A.; Aoki, K.; Watanabe, J.; J. Electroanal. Chem. 1999, 464, 128.

2. Whightman, R.M.; Wipf, D.O. In Eletroanalytical Chemistry; Bard, A.J., eds., Marcel Dekker: New York, 1989, vol. 15, p. 267.

3. Ceballos, C.; Fernández, H.; J. Am. Oil Chem. Soc. 2000, 77, 731.

4. Gao, J.; Njue, C.K.; Mbindyo, J.K.N.; Rusling, J.F.; J. Eletroanal. Chem. 1999, 464, 31.

5. Sjöblom, J.; Lindberg, R.; Friberg, S.E.; Adv. Colloid Interface Sci. 1996, 95, 125.

6. Romero, J.S.E.; Afonso, E.F.S.; Alvarez-Coque, M.G.G.; Ramos, G.R.; Anal. Chim. Acta 1990, 235, 317.

7. Scwuger, M.J.; Stckdorn, K.; Schomäcker, R.; Chem. Rev. 1995, 95, 849.

8. Hellweg, T.; Eimer, W.; Colloids Surf. A 1998, 136, 97.

9. Ortí, J.F.N.; Camañas, R.M.V.; Ramos, G.R.; Anal. Chim. Acta 1999, 387, 127.

10. Radomska, A.; Dobrucki, R.; Int. J. Pharm. 2000, 196, 131.

11. Wennerström, H.; Söderman, O.; Olsson, U.; Lindman, B.; Colloids Surf. A 1997, 123-124, 13.

12. Dantas, T.N.C.; Da Silva, A.C.; Neto, A.A.D.; Fuel 2001, 80, 75.

13. Moulik, S.P.; Digout, L.G.; Alylward, W.M.; Palepu R., Langmuir 2000, 16, 3101.

14. Klier, J.; Tucker, C.J.; Kalantar, T. H.; Green, D.P.; Adv. Mater. 2000, 12, 1751.

15. Dixit, S.G.; Mahadeshwar, A.R.; Haram, S.K.; Colloids Surf. A 1998, 133, 69.

16. Gradzielski, M.; Hoffmann, H.; J. Phys. Chem. 1994, 98, 2613.

17. Charlton, I.D.; Doherty, A.P.; J. Phys. Chem. B 2000, 104, 8061.

18. Watarai, H.; J. Chromatogr. A. 1997, 780, 93.

19. Chang, H.C.; Lin, Y.Y.; Chern, C.S.; Lin, S.Y.; Langmuir 1998, 14, 6632 .

20. Olla, M.; Monduzzi, M.; Ambrosone, L; Colloids Surf. A 1999, 160, 23. 
21. Schelly, Z.A.; J. Mol. Liq. 1997, 72, 3.

22. Olabisi, O.; Robeson, L.M.; Shaw, M.T.; Polymer-Polymer Miscibility, Academic Press: New York, 1979.

23. Myers, S.A.; Mackay, R.A.; Btajter-Toth, A.; Anal. Chem. 1993, 65, 34.

24. Mackay, R.A.; Myers, S.A.; Bodalbhai, L.; Btajter-Toth, A.; Anal. Chem. 1990, 62, 1084.

25. Oldham, H.B; Myland, J.C.; Fundamentals of Electrochemical Science, Academic Press: San Diego, 1994.

26. Bond, A.M.; Analyst 1994, 119, R1.

27. Mc Bride, H.D.; Evans, D.G.; Anal. Chem. 1973, 45, 446.

28. Takamura, K.; Fuse, T.; Kensuke, A.; Kusu, F.; J. Electroanal. Chem. 1999, 468, 53.

29. Tomkins, R.P.T.; Popovych, O.; Nonaqueous Solution Chemistry, John Wiley \& Sons: New York, 1979.

30. Chen, J.; Ikeda, O.; Aoki, K.; J. Electroanal. Chem. 2001, 496,88 .
31. Provencher, S.W.; Makromol. Chem. 1979, 180, 201.

32. Bond, A.M.; Lay, P.A.; J. Electroanal. Chem. 1986, 199, 285.

33. Goldszal, A.; Coasteux, S.; Djabourov, M.; Colloids Surf. A 1996, 112, 141.

34. Claire, K.; Pecora, R.; J. Phys. Chem. B 1997, 101, 746.

35. Cazabat, A.M.; Langevin, D.; J. Chem. Phys. 1981, 74, 3148.

36. Eicke, H.F.; Borkovec, M.; Das-Gupta, B.; J. Phys. Chem. 1989, 93, 314.

37. Robinson, R.A.; Stokes, R.H.; Electrolyte Solutions, $2^{\text {nd }}$ ed., Butterworths \& Co. Ltd. Publishers: London, 1970.

38. Lund, H.; Baizer, M.M.; Organic Electrochemistry, $3^{\text {rd }}$ ed., Marcel Dekker, Inc.: New York, 1990.

39. Przybylski, R.; Zambiazi, R.C.; J. Am. Oil Chem. Soc. 2000, 77, 925.

Received: February 4, 2003 Published on the web: August 14, 2003 